\title{
THE NATURE OF RADIO INTERFERENCE AND SOME LINES OF DEFENSE
}

\author{
J. RICHARD FISHER \\ NRAO, Green Bank, WV 24944 U.S.A.
}

\begin{abstract}
As competition for radio spectrum space continues to increase, radio astronomers can expect to put more technical effort into ways of observing in the presence of interference. Much of the spectrum outside of exclusive radio astronomy frequency bands will continue to be available to the science if receivers and antennas are designed to make efficient use of times, frequencies, directions, and coherence envelopes that do not contain sources of interference. The paper outlines the state of the art in antenna sidelobe reduction, high dynamic range spectrometers, and receiver designs for handling large signals. Techniques for excising pulsed interference on very short timescales and a few thoughts on signal canceling techniques are discussed.
\end{abstract}

\section{INTRODUCTION}

Cooperative use of the radio spectrum means more than frequency allocations and a modicum of geographic isolation. If we think of man-made radio radiation as being distributed in the multidimensional space of time, frequency, polarization, location, direction of arrival, and so forth, and we assume that the radio regulations confine all transmitters to as small a portion of that space as needed for their purposes, how much is left for radio astronomy? Quite a lot, really. For example, the spectrum below $500 \mathrm{MHz}$ is considered to be a poor environment by radio astronomy standards, but a look at just two coordinates of this space, frequency and time, in Fig. 1 shows that a large fraction of the area is free of radiation to the sensitivity of the measurement. If we add two more coordinates to this diagram (direction of arrival), we would see that the celestial hemisphere above a few degrees elevation is virtually free of radio interference in this band. The problem, then, becomes one of rejecting frequencies, times, and/or directions or arrival that contain interference. Since isolation cannot be perfect in any one coordinate, a combination of rejection techniques is usually required.

\section{SPATIAL ISOLATION}

The gain of a 100 -meter antenna at $90 \mathrm{~cm}$ is about $48 \mathrm{~dB}$ over isotropic. From Fig. 2 we can see that this means an isolation of 45 to $60 \mathrm{~dB}$ for directions more than 10 degrees from the main beam of present antennas. A one-Jy radio source has a flux density of $-210 \mathrm{dBW}$ in a $100 \mathrm{kHz}$ band, which is about $73 \mathrm{~dB}$ 
weaker than the weakest interference visible in Fig. 1. The strongest signal in Fig. 1 is about $90 \mathrm{~dB}$ stronger than the one-Jy source. Similar ratios apply to weaker radio sources and weaker interference at higher frequencies.

Antenna sidelobe response could be 10 to $20 \mathrm{~dB}$ lower, but not without a structural redesign. Most of the far sidelobe response comes from scattering from the feed support structure. The sidelobe pattern due to scattering from the feed tripod on the Dwingeloo telescope is shown in Fig. 3. The sidelobe background in this map is about $-60 \mathrm{~dB}$ due to less coherent scattering from the structure. Figure 4 shows what could be achieved without blockage. Compare this with Fig. 2. Most existing radio astronomy antennas are rather poorly designed for far sidelobe suppression, and much of the improvement possible with a fully offset antenna could be achieved with a symmetrical design by making the feed supports much thinner and keeping them farther from the feed. Receivers could be made much lighter, and some imaginative use of distributed dielectric structural components could help a lot.

\section{FREQUENCY ISOLATION}

Since we cannot reject all interference with spatial isolation, radio astronomy receivers must be tolerant of signals that are received. Most receiver front-ends are designed with sensitivity as the overriding criterion. This often means plenty of gain in the first wideband stages. Where high-level interference is a problem, a much closer compromise will need to be struck between bandwidth, noise figure, and gain. More band limiting is needed earlier in the system. Minimum gain at each amplifier stage and lower noise, high-signalhandling amplifiers and mixers are essential.

Spectrometers have to deal with signals in their passband without destroying large portions of their spectrum. Because of its moderate cost, stability, and bandwidth flexibility, the most common spectrometer in use at centimeter and decimeter wavelengths is the autocorrelator. Its frequency response to a narrow band signal with no weighting of the autocorrelation function is shown in Fig. 5, and the envelope of this response is shown on a logarithmic scale by the top curve in Fig. 6. Hanning convolution or autocorrelation function tapering can reduce the wings of this response function by about $10 \mathrm{~dB}$, but experience has been that all but reasonably weak narrow band interference causes trouble to the autocorrelator spectrum.

Filter banks generally have much better channel-to-channel isolation, but they bring back the old problems of stability and the need for many filter banks for different bandwidths. Another approach that shows promise is th FFT spectrometer. Its frequency response envelope, without input weighting, is shown by the middle curve in Fig. 6, and with a moderate weighting function the out-of-channel response could be as low as the bottom curve. The FFT device requires multibit arithmetic and is more expensive than the autocorrelator for 1000 channels or less, but the better interference isolation may make it the spectrometer of choice for work below $5 \mathrm{GHz}$ or so. Computer simulations show that the multiplying element in the FFT must carry about 16 bits fixed-point or 10 or more floating-point mantissa bits to reach 40 to $50 \mathrm{~dB}$ channel-to-channel isolation. 


\section{TIME ISOLATION}

At the frequency of an interfering signal the times that are open for cosmic measurements range in length from microseconds to hours depending on the nature of the interference. Power line noise and radar often have a reasonably low duty cycle, but the pulse spacings can be submillisecond. If a spectrometer or continuum detector can subdivide its data on these timescales, in principle, it can throw out the contaminated pieces.

The task of determining which data to discard can be done with a circuit similar to the one in Fig. 7. In this circuit the slow integrator follows the average receiver power output over many interfering pulses and the fast integrator responds to the fast pulse rise time. When the fast output exceeds the slow output by a selected amount, the data are flagged bad. Without the slow integrator the threshold could not be set very close to the receiver noise fluctuations without tripping on gain drifts and source power changes. The fast integrator speed should match the interfering pulse width for best sensitivity.

One way of excising bad data is to hold the detector level until the interference goes away, but this does not result in the best receiver sensitivity. A better way is to average the good data and divide by the fraction of time containing good data. A test of such a running division scheme is shown in Fig. 8 where the interfering signal was fairly strong ignition noise. Time discrimination longer than a second can be done without special hardware by doing equivalent operations in a computer as long as the data are recorded with enough time resolution.

Even for continuum work, the combined strategy of frequency and time isolation may be warranted for the most difficult interference environments. Something like an FFT spectrometer could find a home in continuum radiometry.

\section{SPECIFIC SIGNAL CANCELLATION}

When there is only one source of interference, specific measures can be taken to cancel the signal from that source. Two schemes come to mind. One uses the fact that all man-made radiation is highly polarized and that the sense of that polarization often changes very slowly. If orthogonally polarized radio telescope outputs are combined with the appropriate relative amplitude and phase, there should exist a resultant elliptical polarization that does not contain the interfering signal. A schematic of such a circuit with adaptive feedback is shown in Fig. 9.

The second scheme is outlined in Fig. 10. Here, an auxiliary antenna with considerably more gain than that of the main antenna's sidelobes is used to sample the interference, and this signal is added to cancel its coherent counterpart in the main receiving system. Similar techniques have been extensively studied under the name of "null steering" in antenna arrays. This technique is particularly difficult for wideband signals because the delay (phase as a function of frequency) needs to be adjusted. Also, for sensitive radiometry the added noise power from the auxiliary antenna must be properly accounted 
for, since it will vary with time as the injection amplitude is varied to track the changes in the interfering signal. Such complex schemes may never find a strong enough need to warrant the effort, but some variation may prove useful.

\section{REFERENCES}

"Sidelobe Performance of Earth-Station Antennas," The Electrical Research Associated, Ltd. Report No. RFTC 301076, June 1978, Surrey, England.

"Interferometric Measurements at $1415 \mathrm{MHz}$ of Radiation Patterns of Paraboloid Antennas at Dwingeloo Radio Observatory," Hartsuiker, A. P., Baars, J. W. M., Dreuth, S., Gelato-Volders, L., IEEE Trans. Ant. and Prop. Vol. Ap-20, 1972, p.166.

"Theory and Application of Digital Signal Processing," Rabiner, L. R. and Gold, B., 1975, Prentice-Hall, Englewood Cliffs, NJ.

"Interference Identification and Excision," A Radio Astronomy Workshop, Green Bank, WV, September 21-22, 1982.

"An Impulse Noise Suppressor for Continuum Radiometry," Morgan, J. V., Jr. and Fisher, J. R., NRAO Electronics Division Internal Report No. 178, Green Bank, WV, Sept. 1977. 


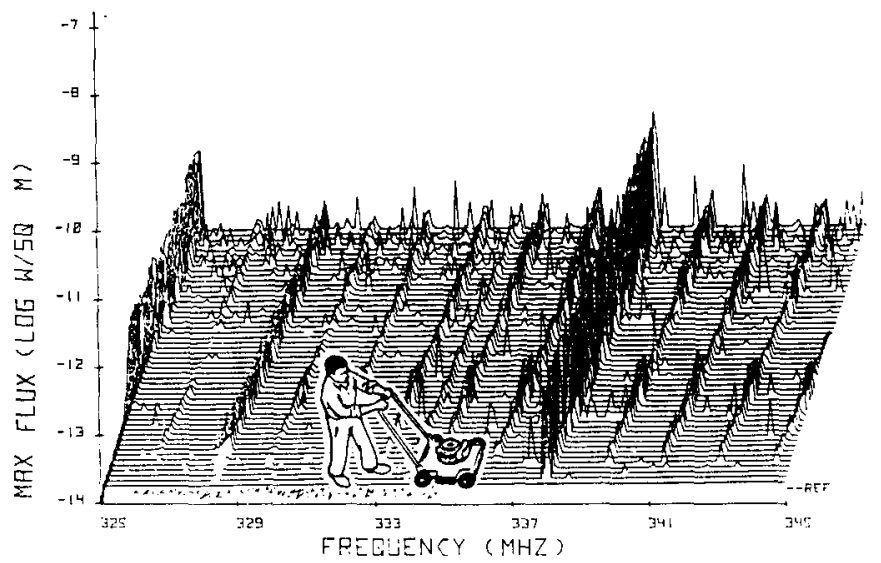

Figure i. Interference intensity vx. Erequency and time from 325 to 345 MHz - part of VLBA site survey. From the cover of Ref. [4].

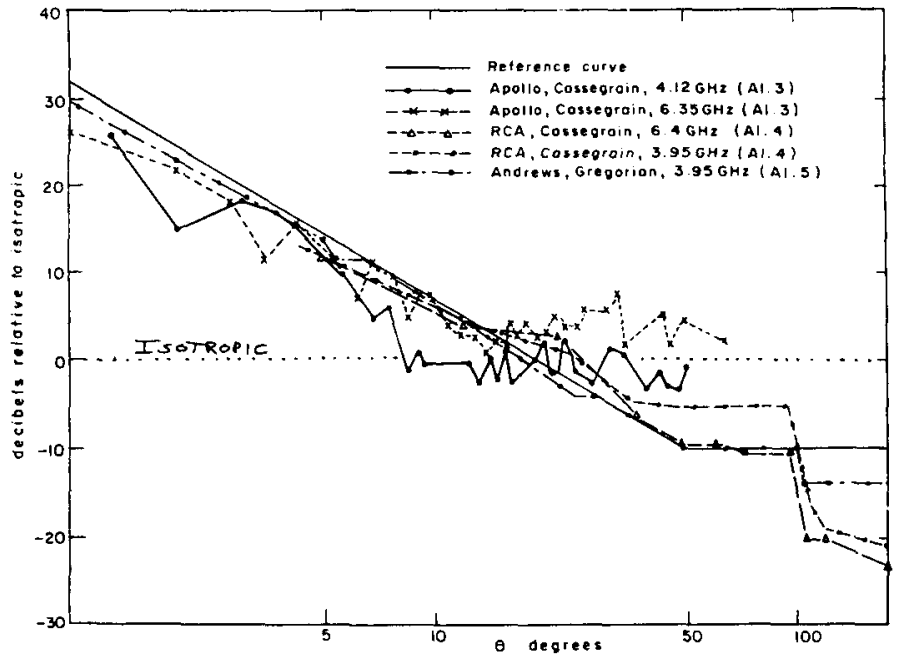

Figure 2. S1delobe envelopes for antennas of diamerar 100 to 300 wavelengths. Ref. [1]. 


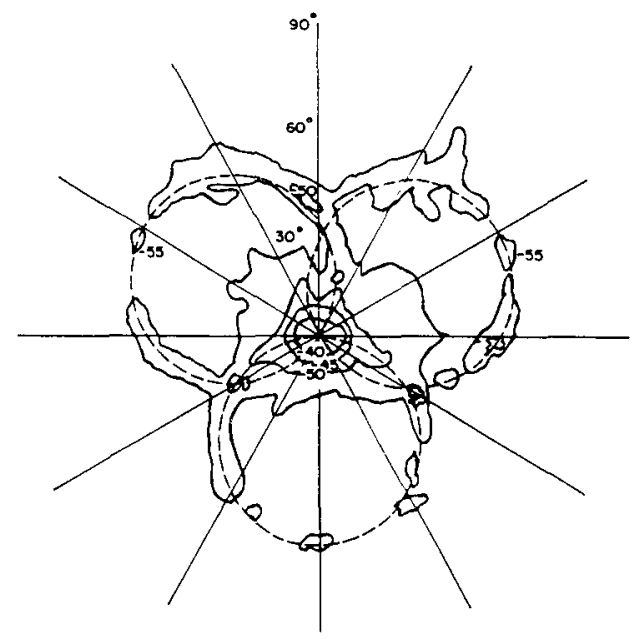

Figure 3. Radiation patcern plot for the Dungeloo 25-m radio telescope at $1415 \mathrm{MHz}$. The dashed circles show the expected positions for the strut scattering sidelobes. Contours are in dB. Ref. [2], Efgure Erom Ref. [1].

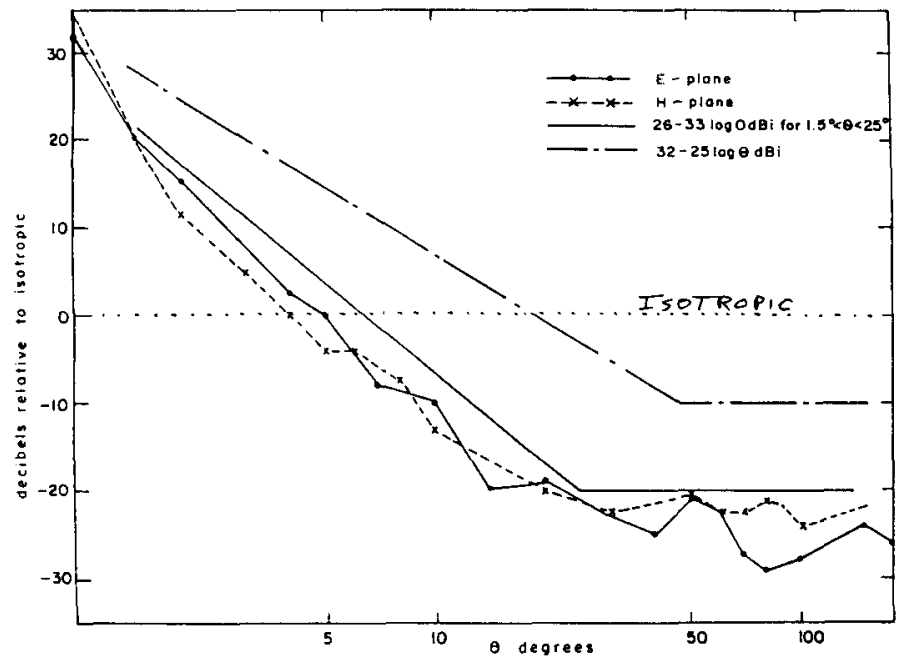

Figure $\rightarrow$, Sideiobe envelope ror an ofiset Gregorian untenna. $\operatorname{Re} \tilde{F}$. [1]. 


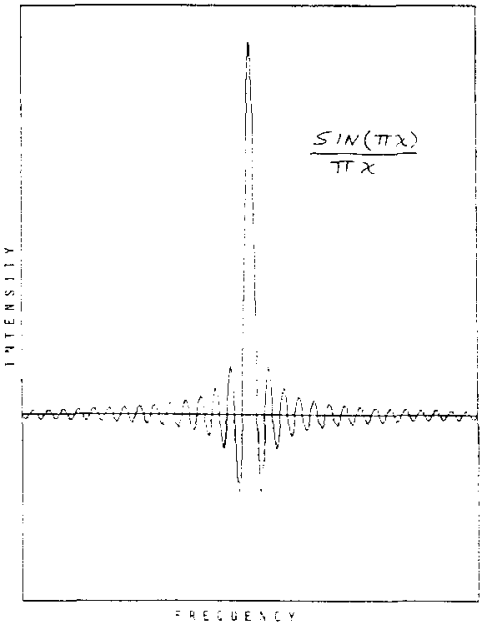

Flgure 5. Instrumental intensity vs. frequency response of an autocorrelation spectromecer with unfform weighting. Linear intanst ty scale.

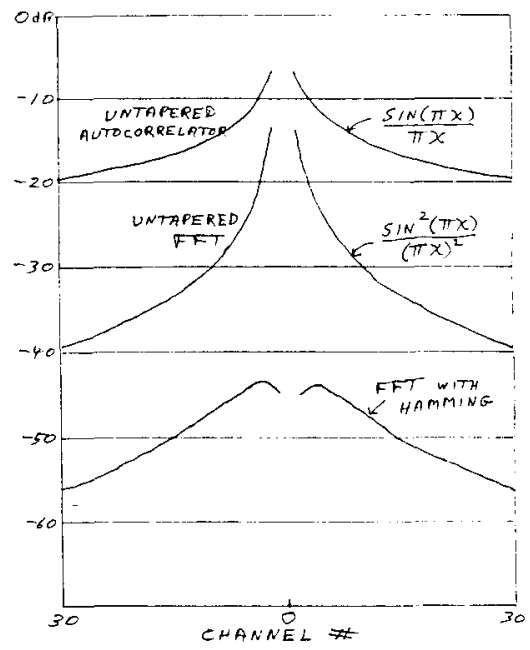

Figure 6. Inteneity vx. f requency responses of an autocorrelation spectrometer with uniform weighting (top curve), of an FFT-type spectrometer with uniform veighting or an autocorrelation spectrometer with ilnear taper veighting autocorrelation spectrometer with linear taper veighting
(middle curve), and of an FFT spectromecer with a Haming saper (bottom curve). Logarithmic intensticy scale. 


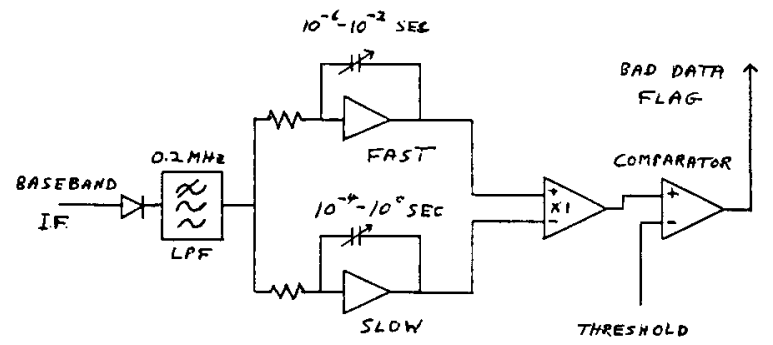

F1gure 7. A uddeband pulse detector with compensation for slow gain and nolse power changes.

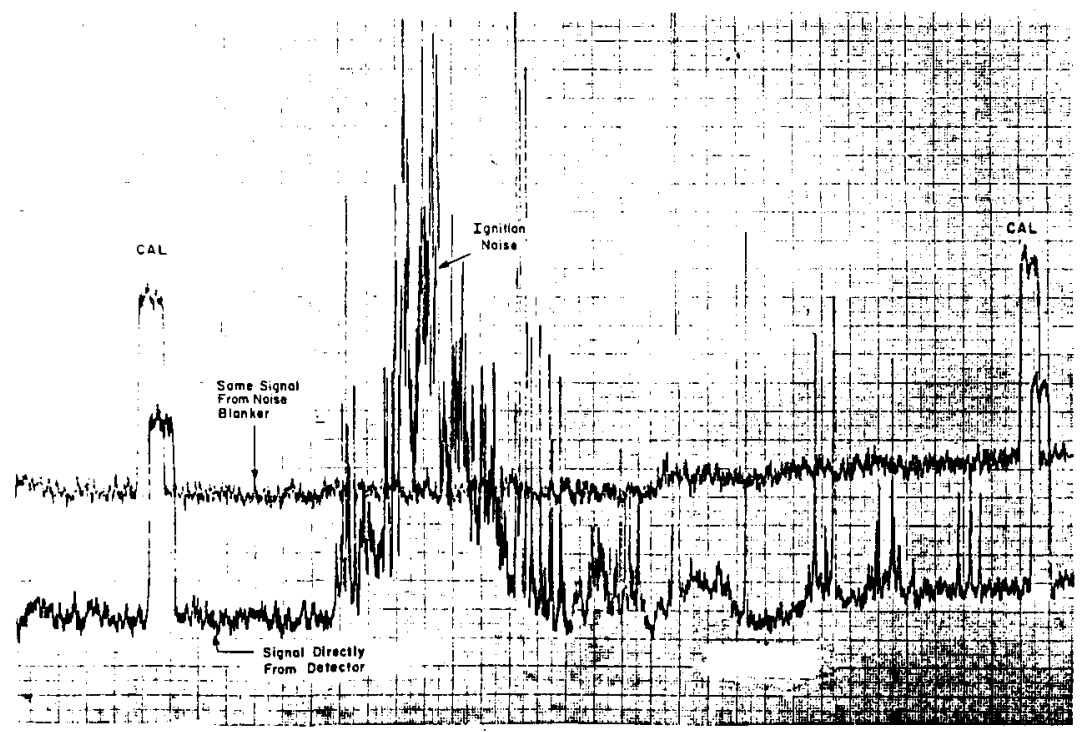

Flgure 8. An example of succestful pulsed notse excistion uaing a puise detector and blanker with a miltiplier to compensate for lost data. Raf. $(5)$. 


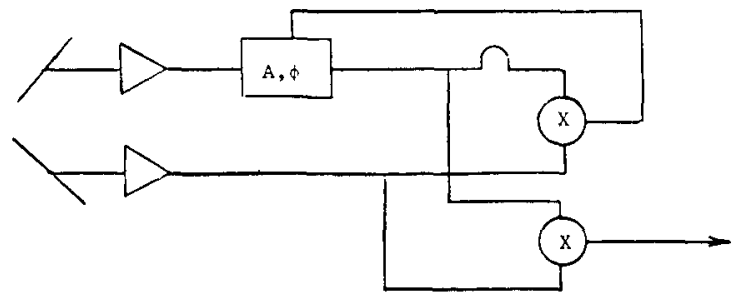

Figure 9. A scheme for libing the partlcular elitiptical polarization that cancalg a mas-made gouroe of ditertarefice.

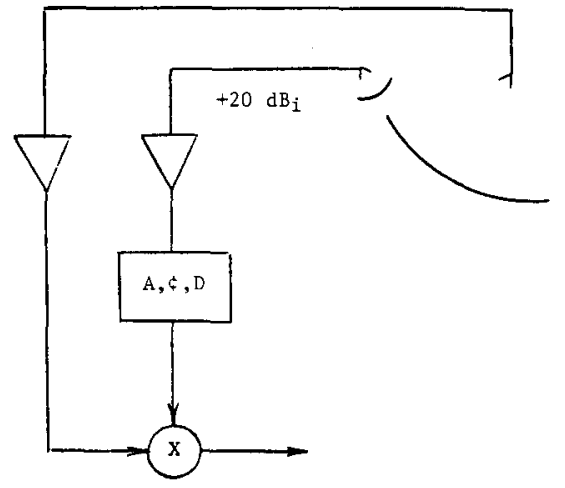

Figure 10. A method for caicolitig an Interferbig siglial in the sldelobe of a radio telescope by selecting the phase, delay, and amplitude or a slgnal frsm an aixlliary antenia. 